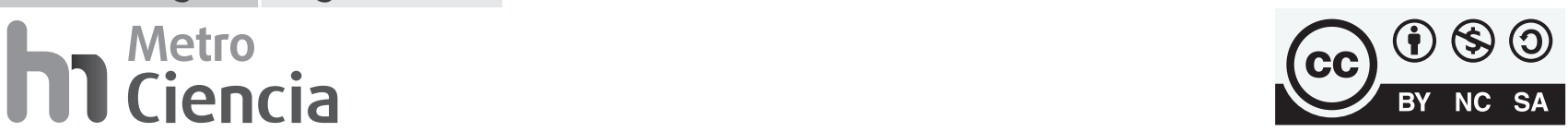

Editorial: Hospital Metropolitano

ISSN (impreso) 1390-2989 - ISSN (electrónico) 2737-6303

Edición: Vol. 29 No 1 (2021) enero-marzo

DOI: https://doi.org/10.47464/MetroCiencia/vol29/1/2021/28-33

URL: https://revistametrociencia.com.ec/index.php/revista/article/view/114

Pág: 28-33

\title{
Hallazgos clínicos en niños con COVID-19 atendidos en el Servicio de Emergencia
}

\section{Clinical findings in children with COVID-19 treated in the Emergency Department}

\author{
Carla Lucía Vaca Yépez' iD, Arelis de Jesús Conde de Vera' iD, \\ Daniel Arturo Espinel Ramos ${ }^{2}$ (D) , Carolina del Valle Aranda Rodríquez ${ }^{1}$ (D), \\ Johanna José Martínez ${ }^{1}$, Diana Carolina Campaña Silva ${ }^{3}$ id \\ Médico Pediatra Tratante, Servicio de Emergencia, Hospital General del Sur de Quito. Ecuador \\ Médico Pediatra, Coordinador Institucional, Servicio de Emergencia Pediátrica, Hospital General del Sur de Quito. Ecuador \\ Médico Residente, Servicio de Emergencia, Hospital General del Sur de Quito. Ecuador
}

Recibido: 01/12/2020 Aceptado: 20/12/2020 Publicado: 29/01/2021

\section{RESUMEN}

Introducción: Los niños han mostrado una menor prevalencia de infección por el virus SARS-CoV-2, así como un curso leve de la enfermedad. Los síntomas que presentan son reflejo de la afectación a nivel respiratorio, digestivo, neurológico y sistémico. Reconocerlos facilita el abordaje de un paciente con sospecha de COVID-19. Métodos: Estudio descriptivo, retrospectivo de tipo transversal, que incluyó 51 niños con infección confirmada por el virus SARS-CoV-2 atendidos en la Emergencia Pediátrica del Hospital General del Sur de Quito durante los meses de abril a junio, con edades entre 1 mes y 17 años 11 meses y 29 días. Los datos se obtuvieron del sistema AS400. El análisis se realizó con el programa estadístico SPSS, se determinaron porcentajes en las variables nominales y medidas de tendencia central y dispersión en las numéricas. Resultados: Se incluyeron 51 niños, la mayoría adolescentes de 10 a 14 años $(29,4 \%)$, y más de la mitad de sexo masculino $(56,9 \%)$. El $82,4 \%$ refirieron manifestaciones clínicas respiratorias. Los síntomas más prevalentes fueron fiebre y tos (68,6\%). La mayoría de pacientes presentaron un curso leve de la enfermedad (60,8\%), pero los menores de un año presentaron enfermedad severa con mayor frecuencia. Conclusiones: Existe un predominio de afectación del sexo masculino. La mayoría acude por síntomas respiratorios y presentan enfermedad leve. Las manifestaciones clínicas más comunes son fiebre y tos.

Palabras claves: SARS-CoV-2, COVID-19, síntomas

\begin{abstract}
Introduction: Children have shown a lower prevalence of SARS-CoV-2 virus infection, as well as a mild course of the disease. The symptoms they present are a reflection of its effect on the respiratory, digestive, neurological and systemic levels. Recognizing them makes it easier to approach a patient with suspected COVID-19. Methods: Descriptive, retrospective, cross-sectional study, which included 51 children with confirmed infection by the SARS-CoV-2 virus treated in the Pediatric Emergency Department of the Hospital General del Sur de Quito during the months of April to June, with ages between 1 month and 17 years 11 months and 29 days. The data was obtained from the AS400 system. The analysis was carried out with the SPSS statistical program, percentages were determined in the nominal variables, whilst measures of central tendency and dispersion were determined in the numerical ones. Results: 51 children were included, most of them adolescents from 10 to 14 years old (29.4\%). More than half were male (56,9\%). 82,4\% reported respiratory clinical manifestations. The most prevalent symptoms were fever and cough (68,6\%). Most of the patients had a mild course of the disease $(60,8 \%)$, but those under one year of age had severe disease more most frequently. Conclusions: There is a predominance of the male sex. Most patients have come with respiratory symptoms and present mild illness. The most common clinical manifestations are fever and cough.
\end{abstract}

Keywords: SARS- CoV-2, COVID-19, symptoms

IDs Orcid

Carla Vaca:

Arelis de Jesús Conde:

Daniel Espinel:

Carolina Aranda:

Johanna Martínez:

Diana Campaña: https://orcid.org/0000-0003-3168-9501 https://orcid org/0000-0003-0068-8282 https://orcid.org/0000-0002-9286-9656 https://orcid.org/0000-0003-4120-087X https://orcid.org/0000-0002-7592-1694 https://orcid.org/0000-0001-7609-5876

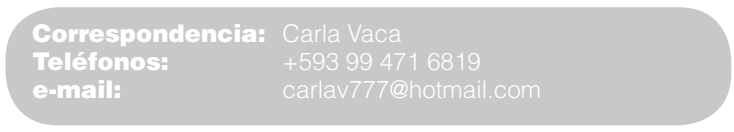




\section{INTRODUCCIÓN}

El coronavirus tipo 2 del Síndrome Respiratorio Agudo Grave (SARS-CoV-2) es el responsable de la enfermedad por coronavirus de 2019 (COVID-19), una infección que ha demostrado una alta patogenicidad y letalidad.

Al 30 de julio, la Organización Mundial de la Salud (OMS) reportó 16.812 .755 casos de COVID-19 alrededor del mundo. En Ecuador la cifra alcanzó un total de 84.370 casos a la misma fecha, de éstos el $4,7 \%$ (3965) correspondió a pacientes con edades comprendidas entre los 0 meses y 19 años ${ }^{1,2}$.

Alrededor del mundo las cifras reportadas de niños con COVID-19 son bajas, sin embargo constituyen una población susceptible a la infección ${ }^{3}$. Los datos publicados revelan que los niños representan el $1-5 \%$ de los casos confirmados de COVID-19, tienen un curso leve de la enfermedad con mejor pronóstico y baja mortalidad ${ }^{4-6}$.

Si bien las manifestaciones clínicas en los niños con COVID-19 son menos severas con relación a las de los adultos, los infantes constituyen un grupo vulnerable, ya que en este grupo etario se han descrito manifestaciones y estadios más severos de la enfermedad $^{7-10}$.

Los niños con COVID-19 pueden estar asintomáticos, o presentar síntomas variables como, fiebre, odinofagia, tos, mialgia, además de síntomas gastrointestinales como diarrea, dolor abdominal, nausea y vómito ${ }^{3,11}$.

Se han publicado además casos de niños con signos y síntomas similares a la enfermedad de Kawasaki ${ }^{12-14}$. En un estudio observacional que incluyó a 21 niños con esta clínica, 76\% presentaron rash polimórfico en la piel, $76 \%$ cambios en los labios y cavidad oral y $81 \%$ inyección conjuntival bulbar bilateral, con la particularidad de que el $95 \%$ presentaron síntomas gastrointestinales consistentes en dolor abdominal, vómito y diarrea ${ }^{15,16}$.

El 15 de mayo del 2020 la OMS alertó sobre el Síndrome Inflamatorio Multisistémico (SIM) que afecta a niños y adolescentes, con un cuadro sugestivo de choque tóxico o características similares a la enfermedad de Kawasaki. En nuestro país han sido reportados un total de 48 casos sospechosos de SIM hasta la semana epidemiológica $29^{17}$.

También han sido descritos como signos al momento de la admisión taquipnea, taquicardia, saturación de oxígeno menor a $92 \%{ }^{18}$.

Respecto a la severidad de la enfermedad, los niños pueden presentar un curso leve, moderado, severo o crítico ${ }^{5,19}$.
Algunos estudios alrededor de mundo han identificado las manifestaciones clínicas en niños, logrando una aproximación diagnóstica y la implementación del tratamiento oportuno ${ }^{11}$.

Conocer la clínica presentada por la población pediátrica en nuestro país permitirá un fácil reconocimiento de los síntomas y signos a los cuales nos podemos enfrentar en el abordaje de un niño con sospecha de COVID-19.

\section{POBLACIÓN Y MÉTODOS}

Se trata de un estudio descriptivo, retrospectivo de tipo transversal, realizado en el servicio de Emergencia Pediátrica del Hospital General del Sur de Quito, Ecuador. El período de estudio se estableció entre el 1ro de abril del 2020 y el 30 de junio del 2020.

La muestra fue conformada por un total de 51 niños con infección por el virus SARS-CoV-2 confirmada por el método de Reacción en Cadena de la Polimerasa con transcripción reversa en tiempo real (RT-PCR) en hisopado nasofaríngeo, con edades comprendidas entre 1 mes y 17 años 11 meses y 29 días.

La muestra fue no probabilística y se incluyeron todos los casos potencialmente elegibles del servicio de Emergencias Pediátricas del Hospital General del Sur de Quito.

Fueron excluidos los niños con edades menores a 1 mes, los niños asintomáticos y los pacientes con datos incompletos en la historia clínica.

Se tomaron en consideración variables demográficas y clínicas: edad, sexo, grupo etario, frecuencia cardiaca, frecuencia respiratoria, saturación de oxígeno, fiebre, manifestaciones sistémicas, respiratorias, digestivas, neurológicas y severidad de la enfermedad.

Los grupos etarios fueron establecidos de acuerdo a los cortes de edad presentados en la infografía de "Situación nacional por COVID-19".

Los rangos normales para los signos vitales fueron tomados de la American Heart Association para cada grupo etario.

La severidad de la enfermedad fue determinada de acuerdo con la clasificación: leve, moderada, severa y crítica descrita por Feng Fang y cols, la Sociedad China de Pediatría y el Comité Editorial de la Revista China de Pediatría.

Enfermedad leve: síntomas de infección aguda del tracto respiratorio superior, como fiebre, tos, odinofagia, rinorrea, estornudos, fatiga y mialgia, sin anormalidades auscultatorias. Algunos niños pueden no 
tener fiebre y solo síntomas digestivos como náuseas, vómitos, dolor abdominal y diarrea.

Enfermedad moderada: signos y síntomas de neumonía, como fiebre, tos productiva predominante y/o sibilancias, pero sin manifestaciones hipóxicas obvias como dificultad para respirar, disnea u otros signos de insuficiencia respiratoria. Algunos casos pueden no tener signos o síntomas clínicos, pero con hallazgos positivos en la tomografía de tórax.

Enfermedad severa: fiebre, tos y disnea (asociada con cianosis central y saturación de oxígeno <92\%), taquipnea o diarrea severa, signos de dificultad respiratoria, como jadeo, retracción, bradipnea y, raramente, apnea.

Enfermedad crítica: progresión a Síndrome de Dificultad Respiratoria Agudo (SDRA) o insuficiencia respiratoria, pudiendo presentar coagulación intravascular diseminada, shock, encefalopatía, miocarditis, insuficiencia cardíaca y enfermedad renal aguda.

Los datos fueron recopilados de la Historia Clínica Única del sistema AS400 del Instituto Ecuatoriano de Seguridad Social y fueron registrados en una matriz, en la que constaron las variables de estudio.

El análisis de datos fue realizado con el programa estadístico informático IBM SPSS statistics versión 25. En el análisis estadístico univariado se determinó porcentajes en las variables nominales y medidas de tendencia central y dispersión en las numéricas.

\section{RESULTADOS}

Fueron incluidos un total de 51 niños y niñas atendidos durante los meses de abril a junio del 2020 en el servicio de Emergencia Pediátrica del Hospital General del Sur de Quito con diagnóstico de infección por el virus SARS- CoV-2 confirmada por el método de Reacción en Cadena de la Polimerasa con transcripción reversa en tiempo real (RT-PCR) en hisopado nasofaríngeo. Los casos no incluidos en el estudio se presentan en la figura 1.

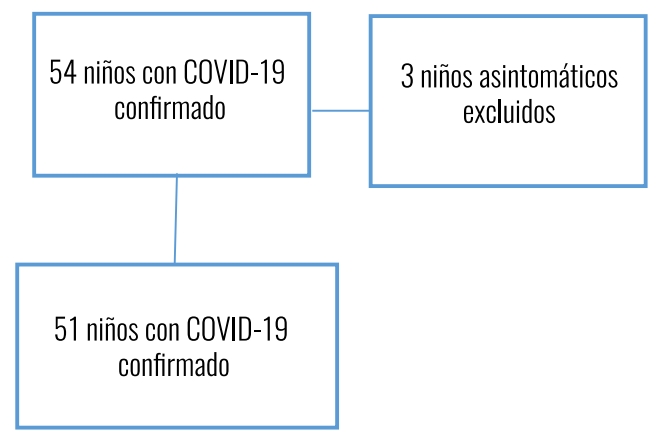

Figura 1. Diagrama de flujo de los participantes del estudio.
Existió un predominio de pacientes de sexo masculino 56,9\% $(n=29)$, en relación con el 43,1\% $(n=22)$ de sexo femenino.

El promedio de edad fue de 108 meses (DE $\pm 69,6)$. El rango de edad fue de 2 meses a 215 meses (17 años 11 meses). El mayor porcentaje de pacientes correspondió al grupo con edades comprendidas entre 10 y 14 años 29,4\% (n=15). (Tabla 1)

Tabla 1. Distribución de pacientes por grupo etario.

\begin{tabular}{lcc} 
& Frecuencia & Porcentaje \\
\hline Menores de un año & 6 & 11,8 \\
De 1 a 4 años & 9 & 17,6 \\
De 5 a 9 años & 11 & 21,6 \\
De 10 a 14 años & 15 & 29,4 \\
De 15 a 17 años & 10 & 19,6 \\
Total & 51 & 100,0 \\
\hline
\end{tabular}

Fuente: Sistema AS400 hospital del IESS Quito Sur, abril-junio 2020.

Del total de pacientes, solamente tres $(5,6 \%)$ tuvieron comorbilidades subyacentes.

El 82,4\% $(n=42)$ presentaron síntomas respiratorios y el $17,6 \%(n=9)$ otro tipo de sintomatología.

Con relación a los signos vitales registrados al momento de la atención se evidenció que, el 76,5\% ( $n=$ 39) tuvieron una frecuencia cardíaca y respiratoria en rango normal para la edad, y más de la mitad de pacientes $56,9 \%(n=29)$ tuvieron una saturación de oxígeno igual o mayor al 94\%. (Tabla 2)

Tabla 2. Signos vitales al ingreso.

\begin{tabular}{llcc} 
& & Recuento & Porcentaje \\
\hline \multirow{2}{*}{$\begin{array}{c}\text { Frecuencia } \\
\text { cardiaca }\end{array}$} & Normal & 39 & $76,5 \%$ \\
& Taquicardia & 12 & $23,5 \%$ \\
& Bradicardia & 0 & $0,0 \%$ \\
\hline \multirow{2}{*}{$\begin{array}{c}\text { Frecuencia } \\
\text { respiratoria }\end{array}$} & Normal & 39 & $76,5 \%$ \\
& Bradipnea & 12 & $23,5 \%$ \\
\hline \multirow{2}{*}{$\begin{array}{c}\text { Saturación } \\
\text { de oxígeno }\end{array}$} & Igual o mayor a 94\% & 0 & $0,0 \%$ \\
& Igual o menor a 89\% & 29 & $56,9 \%$ \\
\hline
\end{tabular}

Fuente: Sistema AS400 hospital del IESS Quito Sur, abril-junio 2020.

Los síntomas más prevalentes fueron fiebre y tos, presentes en el 68,6\% ( $n=35)$ de pacientes. Mientras que, a nivel sistémico, respiratorio, digestivo y neurológico fueron encontradas otras manifestaciones clínicas menos frecuentes. (Tabla 3) 
Tabla 3. Manifestaciones clínicas.

\begin{tabular}{llrr} 
& & Recuento & Porcentaje \\
\hline \multirow{3}{*}{ Sistémico } & Fiebre & $35 / 51$ & $68,6 \%$ \\
& Mialgia & $3 / 51$ & $5,9 \%$ \\
& Artralgia & $3 / 51$ & $5,9 \%$ \\
& Dermatosis & $1 / 51$ & $2,0 \%$ \\
\hline \multirow{3}{*}{ Respiratorio } & Rinorrea & $10 / 51$ & $19,6 \%$ \\
& Anosmia & $3 / 51$ & $5,9 \%$ \\
& Odinofagia & $14 / 51$ & $27,5 \%$ \\
& Tos & $35 / 51$ & $68,6 \%$ \\
& Dolor en el pecho & $3 / 51$ & $5,9 \%$ \\
\hline \multirow{3}{*}{ Digestivo } & Ageusia & $1 / 51$ & $2,0 \%$ \\
& Vómito & $6 / 51$ & $11,8 \%$ \\
& Diarrea & $8 / 51$ & $15,7 \%$ \\
& Dolor abdominal & $3 / 51$ & $5,9 \%$ \\
\hline \multirow{2}{*}{ Neurológico } & Cefalea & $11 / 51$ & $21,6 \%$ \\
& Convulsiones & $3 / 51$ & $5,9 \%$ \\
\hline
\end{tabular}

Fuente: Sistema AS400 hospital del IESS Quito Sur, abril-junio 2020
En cuanto a la severidad de la enfermedad, la mayoría de pacientes presentaron un curso leve 60,8\% $(n=31)$ y solamente hubo un paciente crítico, el cual falleció. (Tabla 4)

Tabla 4. Severidad de la enfermedad.

\begin{tabular}{rlrr} 
& & Recuento & Porcentaje \\
\hline \multirow{4}{*}{ Severidad } & 31 & 60,8 \\
& Leve & 11 & 21,6 \\
& Moderado & 8 & 15,7 \\
& Severo & 1 & 2,0 \\
& Crítico & 51 & 100,0 \\
\hline
\end{tabular}

Fuente: Sistema AS400 hospital del IESS Quito Sur, abril-junio 2020.

El grupo etario que concentró al mayor número de pacientes con enfermedad severa correspondió al de menores de un año, mientras que el paciente que presentó un curso crítico fue un adolescente de 15 años. (Tabla 5)

De los pacientes atendidos, el 5,9\% $(n=3)$ fueron ingresados en UCIP, dos de ellos ameritaron cuidados intermedios y uno ventilación mecánica.

Tabla 5. Severidad de la enfermedad por grupo etario.

\begin{tabular}{|c|c|c|c|c|c|c|c|c|c|}
\hline & & \multicolumn{8}{|c|}{ Severidad } \\
\hline & & \multicolumn{2}{|c|}{ Leve } & \multicolumn{2}{|c|}{ Moderado } & \multicolumn{2}{|c|}{ Severo } & \multicolumn{2}{|c|}{ Crítico } \\
\hline & & Recuento & $\%$ & Recuento & $\%$ & Recuento & $\%$ & Recuento & $\%$ \\
\hline \multirow{5}{*}{$\begin{array}{l}\text { Grupo } \\
\text { etario }\end{array}$} & Menores de un año & 1 & $2,0 \%$ & 2 & $3,9 \%$ & 3 & $5,9 \%$ & 0 & $0,0 \%$ \\
\hline & De 1 a 4 años & 6 & $11,8 \%$ & 2 & $3,9 \%$ & 1 & $2,0 \%$ & 0 & $0,0 \%$ \\
\hline & De 5 a 9 años & 8 & $15,7 \%$ & 1 & $2,0 \%$ & 2 & $3,9 \%$ & 0 & $0,0 \%$ \\
\hline & De 10 a 14 años & 12 & $23,5 \%$ & 3 & $5,9 \%$ & 0 & $0,0 \%$ & 0 & $0,0 \%$ \\
\hline & De 15 a 17 años & 4 & $7,8 \%$ & 3 & $5,9 \%$ & 2 & $3,9 \%$ & 1 & $2,0 \%$ \\
\hline
\end{tabular}

Fuente: Sistema AS400 hospital del IESS Quito Sur, abril-junio 2020.

\section{DISCUSIÓN}

El presente estudio demostró un mayor predominio de la enfermedad en pacientes de sexo masculino (56,9\%), un resultado similar al descrito en otros estudios como el análisis clínico de Ke Bai y cols., que incluyó a 25 pacientes de los cuales el 56\% fueron masculinos o el metaanálisis de Vasco y cols., con $56 \%$ varones ${ }^{20,21}$.

Las manifestaciones clínicas vistas con mayor frecuencia en los niños son fiebre y tos seca ${ }^{22-25}$.

En el estudio el $68,6 \%$ de los pacientes presentaron fiebre y tos. Resultados que han sido descritos en otros estudios como en el de Zhen et al., quienes reportaron fiebre en el $52 \%$ y tos $44 \%$ de los 25 pacientes incluidos. Chang et al., en su revisión sistemática hallaron como síntoma más prevalente la fiebre $59 \%$ seguido de tos $46 \%$; Du et al., reportaron fiebre en el $35,7 \%$ y tos en el $21,4 \%$ de los 14 niños incluidos en su estudio $22-25$.
Han sido reportados además síntomas menos frecuentes como congestión nasal, síntomas gastrointestinales, fatiga, cefalea ${ }^{26}$.

En este estudio, en orden de frecuencia se encontraron: odinofagia $27,5 \%$, cefalea $21,6 \%$, rinorrea $19,6 \%$, diarrea $15,7 \%$ y vómito $11,8 \%$.

En una revisión del Centro Chino para el Control y Prevención de Enfermedades de un total de 171 confirmados, el $48,5 \%$ presentó tos, $46,2 \%$ eritema faríngeo, 41,5\% fiebre, 8,8\% diarrea, 7,6\% fatiga, $7,6 \%$ rinorrea, $6,4 \%$ vómito, $5,3 \%$ congestión nasal ${ }^{18}$.

Las manifestaciones gastrointestinales son más frecuentes en niños que en adultos, entre estas se mencionan dolor abdominal, nausea, vómito, diarrea, anorexia que en ocasiones pueden crear confusión con cuadros de apendicitis aguda ${ }^{10,27}$.

En un estudio que incluyó 244 casos confirmados, se determinó que 193 fueron sintomáticos, 
de éstos 34 (17,7\%) presentaron manifestaciones digestivas que, en orden de frecuencia fueron: vómito $(9,47 \%)$, diarrea $(6,17 \%)$, anorexia $(3,29 \%)$ y dolor abdominal $(1,61 \%)^{28}$. Mientras que, en la presente investigación los síntomas digestivos encontrados fueron diarrea en el $15,7 \%$ de los niños, seguido de vómito $11,8 \%$, dolor abdominal $5,9 \%$ y ageusia $2 \%$.

Además, han sido publicados casos de niños con lesiones dérmicas como son erupciones similares a micosis en zonas acrales, lesiones papulo-vesiculares similares a las de varicela, exantema urticarial, morbiliforme y petequial ${ }^{29-31}$. En el estudio solamente un niño presentó dermatosis.

Con relación a los signos clínicos descritos con mayor frecuencia destacan eritema faríngeo, taquicardia y taquipnea al ingreso. En la revisión sistemática de Souza y cols. de los 393 casos de niños con COVID-19 en los cuales fueron descritas las manifestaciones clínicas se encontró que el 20,6\% ( $n=81)$ presentaron eritema faríngeo, 18,6\% $(n=73)$ taquicardia y $13,4 \%(n=53)$ taquipnea ${ }^{26}$.

El estudio reveló taquicardia en el 23,5\%, taquipnea en el $23,5 \%$ de pacientes y saturación de oxígeno igual o menor a $89 \%$ en el $11,8 \%$.

La severidad de la enfermedad ha sido clasificada en función de los síntomas, signos y hallazgos de imagen presentados por los pacientes.

En la Guía de "Manejo clínico de la infección respiratoria aguda grave causada por el nuevo coronavirus (2019-nCoV)" de la OMS, se menciona que el curso clínico de la infección puede ser leve, moderado o grave, contemplando en este último caso a los pacientes con neumonía, síndrome de dificultad respiratoria aguda (SDRA), septicemia y choque séptico ${ }^{32}$.

Fang et al, propusieron la clasificación de la enfermedad en: infección asintomática, infección leve, moderada, grave y crítica en función de las características clínicas que presentaron los casos pediátricos con diagnóstico establecido de COVID-1933.

Con relación a la severidad de la enfermedad Dong y cols., encontraron que de los 728 casos confirmados $43,1 \%$ fueron leves, $40,9 \%$ moderados, $2,5 \%$ severos y $0,4 \%$ críticos $^{9}$.

Mientras que en la revisión sistemática de Souza y cols., que incluyó a 1117 casos, se halló que el 14\% fueron asintomáticos, $36,3 \%$ presentaron un curso leve de la enfermedad, $46 \%$ moderado, $2,1 \%$ severo y $1,2 \%$ crítico $^{26}$.
En el presente estudio el $60,8 \%$ de niños presentaron un curso leve de la enfermedad, 21,6\% moderado, $15,7 \%$ severo y $2 \%$ crítico.

Son muchos los estudios que han sido publicados sobre COVID-19 en un corto período de tiempo, sin embargo, todavía hay mucho que aprender sobre el impacto de esta patología en los niños, sobre todo a nivel local.

\section{RECOMENDACIONES}

Se recomienda una actualización continua sobre la incidencia, prevalencia y curso clínico de la enfermedad en niños en el país, lo que nos permitirá realizar comparaciones con la evidencia disponible a nivel mundial.

\section{CONCLUSIONES}

El estudio mostró un predominio de afectación del sexo masculino y de adolescentes con edades comprendidas entre 10 y 14 años. La mayoría de pacientes presentan un curso leve de la enfermedad. Los síntomas respiratorios son el principal motivo de consulta. Las manifestaciones clínicas más comunes son fiebre y tos, mientras que manifestaciones menos frecuentes a nivel sistémico, digestivo, respiratorio y neurológico pueden estar presentes. Los lactantes representan un grupo susceptible y con mayor riesgo de presentar formas severas de la enfermedad.

\section{CONTRIBUCIÓN DE LOS AUTORES}

CV: Concepción y diseño del trabajo; recolección y obtención de resultados; análisis e interpretación de datos; redacción del manuscrito.

AdJC: Concepción y diseño del trabajo; recolección y obtención de resultados; análisis e interpretación de datos.

DE: Concepción y diseño del trabajo; recolección y obtención de resultados; análisis e interpretación de datos.

CA: Concepción y diseño del trabajo; recolección y obtención de resultados; análisis e interpretación de datos.

JM: Concepción y diseño del trabajo; recolección y obtención de resultados.

DC: Concepción y diseño del trabajo; recolección y obtención de resultados.

\section{CONFLICTOS DE INTERÉS}

Los autores declaran no tener conflictos de interés. 


\section{FINANCIAMIENTO}

Los autores realizaron el financiamiento de los gastos incurridos en la producción de este artículo.

\section{AGRADECIMIENTOS}

Se reconoce el trabajo del personal del Servicio de Emergencias del Hospital General del Sur de Quito.

\section{REFERENCIAS BIBLIOGRÁFICAS}

1. MSP. El MSP informa: Situación coronavirus Covid-19 (10-09-2020) [Online].; 2020 [cited 202103 17. Available from: https://www.salud. gob.ec/el-ministerio-de-salud-publica-del-ecuador-msp-informa-situacion-coronavirus/

2. WHO. Coronavirus disease (COVID-19): situation report, 151. Ginebra, Suiza: World Health Organization; 2020

3. Hong H, Wang Y, Chung HT, Chen CJ. Clinical characteristics of nove coronavirus disease 2019 (COVID-19) in newborns, infants and children. Pediatric and Neonatology. 2020 Marz; 61(2): 131-132.

4. Bialek S, Gierke R, Hughes M, McNamara LA, Pilishvili T, Skoff T. Coronavirus disease 2019 in children - United States, February 12-Apri 2, 2020. Morbidity and Mortality Weekly Report. 2020 Abr; 69(14)

5. Ludvigsson JF. Systematic review of COVID-19 in children shows milder cases and a better prognosis than adults. Acta Paediatrica. 2020 Mar; 109(6): 1088-1095.

6. WHO. Report of the WHO-China Joint Mission on Coronavirus Disease 2019 (COVID-19). Ginebra, Suiza: World Health Organization; 2020

7. Allana A, Aziz Ali S, Faisal Saleem A. The clinical spectrum of COVID-19 in neonates and infants: A systematic review protocol. Research Square. 2020 May; 31229(1).

8. Jiehao C, Jin X, Daojiong L, Zhi Y, Lei X. A Case Series of Children With 2019 Novel Coronavirus Infection: Clinical and Epidemiological Features. Clinical Infectious Diseases. 2020 Sep; 71(6): 1547-1551.

9. Dong $Y, M o X, H u$ Y, Qi X. Epidemiology of COVID-19 Among Children in China. PEDIATRICS. 2020 Jun; 145(6): 1-12

10. Zimmermann P, Curtis N. Coronavirus Infections in Children Including COVID-19: An Overview of the Epidemiology, Clinical Features, Diagnosis, Treatment and Prevention Options in Children. Pediatr Infect Dis J. 2020 May; 39(5): p. 355-368

11. Qiu H, Wu J, Hong L, Luo Y. Clinical and epidemiological features of 36 children with coronavirus disease 2019 (COVID-19) in Zhejiang, China: an observational cohort study. The Lancet Infectious Diseases. 2020 Jun; 20(6): 689-696

12. Jones VG, Mills M, Suarez D, Hogan CA. COVID-19 and Kawasak Disease: Novel Virus and Novel Case. Hospital Pediatrics. 2020 Jun 10(6): 537-540.

13. Riphagen S, Gomez X, Gonzalez-Martinez C. Hyperinflammatory shock in children during COVID-19 pandemic. The Lancet. 2020 May; 395(10237): 1607-1608.

14. Schroeder AR, Wilson KM, Ralston SL. COVID-19 and Kawasak Disease: Finding the Signal in the Noise. Hosp Pediatr. 2020 Oct; 10(10): e1-e3.

15. Morand A, Urbina D, Fabre A. COVID-19 and Kawasaki Like Disease: The Known-Known, the Unknown-Known and the Unknown-Unknown. Preprints. 20320 May; 160(1)
16. Toubiana J, Poirault C, Corsia A, Bajolle F, Fourgeaud, Angoulvant F, et al. Kawasaki-like multisystem inflammatory syndrome in children during the covid-19 pandemic in Paris, France: prospective observational study. The bmj. 2020 Apr; 369(m2094)

17. MSP. Boletines Síndrome Inflamatorio multisistémico (SIM) en niños y adolescentes menores de 19 años asociado a COVID-19. Quito, Ecuador: Subsecretaria Nacional de Vigilancia de la Salud Pública; 2020 .

18. Lu X, Zhang L, Du H, Zhang J, Li YY, Qu J. SARS-CoV-2 Infection in Children. N Engl J Med. 2020; 382(1): 1663-1665.

19. Panzeri Carlotti APdC, Brunow de Carvalho W, Johnston C. COVID-19 Diagnostic and Management Protocol for Pediatric Patients. Clinics (Sao Paulo). 2020 Apr; 75(1)

20. Bai K, Wenjun L, Chengjun L, Yueqiang F, Jun H. Clinical Analysis of 25 COVID-19 Infections in Children. The Pediatric Infectious Disease Journal. $2020 \mathrm{Jul}$; 39(7)

21. Vasco-Morales S, Vasco-Toapanta C, Toapanta-Pinta. Características clínicas, radiológicas y de laboratorio en niños con diagnóstico de COVID-19: Metaanálisis de proporción única. Scielo Preprint. 2021.

22. Chang TH, Wub JL. Clinical characteristics and diagnostic challenges of pediatric COVID-19: A systematic review and meta-analysis. Journal of the Formosan Medical Association. 2020 May; 119(5): 982-989.

23. Du W, Yu J, Wang H, Zhang X, Qiang Li SZ, Zhang Z. Clinical characteristics of COVID-19 in children compared with adults in Shandong Province, China. Infection. 2020 Apr; 48(1): 445-452.

24. Ma H, Hu J, Tian J, Zhou X, Li H. A single-center, retrospective study of COVID-19 features in children: a descriptive investigation. BMC Med. 2020 May; 18(1): 123-126

25. Zheng F, Liao C, Jin Rm. Clinical Characteristics of Children with Coronavirus Disease 2019 in Hubei, China. Current Medical Science. 2020; 40(1): 275-280.

26. De Souza TH, Nadal JA, Nogueira RJ. Clinical manifestations of children with COVID-19: A systematic review. Pediatric Pulmonology. 2020 Ago; 55(8): 1892-1899

27. Tullie L, Ford K, Bisharat M, Watson T. Gastrointestinal features in children with COVID-19: an observation of varied presentation in eight children. The Lancet Child \& Adolescent Health. 2020 May; 4(7): 1920

28. Xiong XI, Kak-yuen Wong K, Chi Sq, Zhou Af, Jian-qiao T. Are COVID-19 infected children with gastrointestinal symptoms different from those without symptoms? A comparative study of the clinical characteristics and epidemiological trend of 244 pediatric cases from Wuhan. MedRxiv preprint. 2020

29. Colonna C, Monzani NA, Rocchi A. Chilblain-like lesions in children following suspected COVID-19 infection. Pediatric Dermatology. 2020 Jun; 37(3): 437-440

30. Genovese G, Colonna C, Marzano AV. Varicellalike exanthem associated with COVID-19 in an 8 year old girl: A diagnostic clue? Pediatric Dermatology. 2020 Jun; 37(3): 435-436.

31. Torrel A, Andina D, Santonja C, Noguera-Morel L, Bascuas-Arribas M. Erythema multiforme-like lesions in children and COVID-19. Pediatr Dermatol. 2020 May; 37(3): 442-446.

32. WHO. Manejo clínico de la infección respiratoria aguda grave presuntamente causada por el nuevo coronavirus (2019-nCoV) Ginebra, Suiza: Organización Mundial de la Salud; 2020.

33. Fang F, Chen Y, Zhao, Liu T, Huang Y, Qiu L, et al. Recommendations for the Diagnosis, Prevention, and Control of Coronavirus Disease-19 in Children-The Chinese Perspectives. Front. Pediatr. 2020 Nov; 8(553394): 16. 